Conclusion. The re- audit was highlighted to inpatient managers, nursing staff, The Medicines Management Committee (MMC) anddoctors in the Learning Disability division. Prescribers werereminded of the importance of documenting a stop date for the prescriptions and signing off once drug is crossed out. It was discussed in MMC to consider removing the standard for recording allergies in red ink as the box is already red in colour. The PRN section for medication does not have an area to sign when the drug is cancelled and this in particular is the case when PRN medication is re-written. It was discussed to limit this standard to regular medication and to be taken in consideration if the current drug chart requires redesigning in the future. We also recommended a re- audit in 2 years' time.

\section{Service evaluation of the mental health assessment service (MHAS) in Dudley, West Midlands}

Mark Winchester ${ }^{\star}$, Amitav Narula, Rachel Davis and Ella McGowan

Dudley and Walsall Mental Health Partnership NHS Trust ${ }^{*}$ Corresponding author.

doi: 10.1192/bjo.2021.943

Aims. To assess how well MHAS meets the service specification

To ascertain areas of good practice

To examine whether the referral form is being used in an appropriate manner

To elucidate areas of good communication and whether any improvement can be made

Background. Launched in 2012, MHAS is the single point of access service for mental health services for patients aged 16-65 years, with a general practitioner (GP) in Dudley, who are not currently open to secondary care. Assessments are completed by a medic, community psychiatric nurse or jointly. It aims to identify the most appropriate care pathway for patients. This audit was a comprehensive assessment of how effective MHAS is at ensuring patients are adequately triaged.

Method. 10 cases from each month between April 2018 and March 2019 were randomly selected from all 980 anonymised MHAS referrals. A proforma was developed based on current practice, previous audits and service specification. A team of four doctors assisted in the data collection and only electronic health records (EHR) were reviewed.

Result. $88.3 \%$ of referrals were recorded on the EHR. Only $61.7 \%$ of referrals used the proforma with the other referrals mostly being in the form of a letter, which often missed out information vital to the triaging process. Only $4.2 \%$ of referrals are from Primary Care Mental Health Nurses (PCMHN) with $85.8 \%$ arising from GPs. Urgent referrals were not discussed with MHAS via telephone contact in about $60 \%$ of cases. The majority of patients had telephone screening completed the same day and were then discussed the next working day at the daily referral meeting. Although a brief summary for the GP was being sent the same day in all cases, over half of the comprehensive assessments were not being sent within the five day timeframe.

Conclusion. All referrals must be uploaded to the EHR and completed using the service's proforma. PCMHNs may be currently under-utilised or effectively doing their jobs at managing mental health patients in primary care. GPs regularly referring via letter require further training and support to use the proforma. The proforma may require simplification to make it easier to complete. The service specification requires review as it makes unrealistic demands of the service. All referrals must be discussed at the daily referral meeting. Further investigation is required to understand why MHAS is struggling to meet timeframes for appointments and letters.

\section{Fruit and vegetable intake among psychiatric inpatients: an electronic health record-based study}

Adelaide Yue ${ }^{1 *}$, Aida Seyedsalehi² ${ }^{2}$ Jonathan Lewis ${ }^{3}$, Konstantinos Ioannidis ${ }^{4}$ and Julia Deakin ${ }^{3}$

${ }^{1}$ School of Clinical Medicine, University of Cambridge; ${ }^{2}$ Department of Public Health and Primary Care, University of Cambridge, Cambridge and Peterborough NHS Foundation Trust; ${ }^{3}$ Cambridge and Peterborough NHS Foundation Trust and ${ }^{4}$ Cambridge and Peterborough NHS Foundation Trust, Department of Psychiatry, University of Cambridge, Faculty of Health Medicine and Life Sciences, University of Maastricht ${ }^{\star}$ Corresponding author.

doi: 10.1192/bjo.2021.944

Aims. Psychiatric illness is associated with premature mortality, which is largely attributable to physical health conditions. Low fruit and vegetable intake is a risk factor for cardiovascular disease, which contributes significantly to this disparity in physical health. This study used routinely collected data from electronic health records to assess fruit and vegetable intake among psychiatric inpatients across a UK mental health trust.

Method. We conducted an anonymised search of de-identified electronic patient records from the Cambridgeshire and Peterborough NHS Foundation Trust (CPFT) research database. We collected data on ICD-10 diagnosis and fruit and vegetable intake for patients aged 18 years or over, with a recorded ICD-10 psychiatric diagnosis, admitted to CPFT inpatient facilities between March 2013 and January 2019 inclusive $(n=$ 1031). Information on fruit and vegetable intake is recorded as part of a General Health and Lifestyle questionnaire, routinely performed within a week of admission. Fruit and vegetable intake in different ICD-10 diagnostic categories was compared using a one-way ANOVA.

Result. Among patients for whom data on fruit and vegetable intake was recorded $(n=768)$, the prevalence of low fruit and vegetable intake (defined as $<5$ portions/day) was $75.9 \%$, and mean fruit and vegetable intake was 2.85 portions/day (95\% CI 2.72-2.98). Fruit and vegetable intake was lowest among patients with schizophrenia (mean $=2.3$ portions/day), significantly worse than other diagnostic groups. In patients with schizophrenia, prevalence of low fruit and vegetable intake was $86.5 \%$.

Conclusion. Low fruit and vegetable intake is common among CPFT psychiatric inpatients, particularly those with schizophrenia. Interventions to improve dietary habits, such as increasing tailored for individuals with psychiatric illness may help to reduce the risk of physical illness. 